\title{
Die TIDieR Checkliste und Anleitung - ein Instrument für eine verbesserte Interventionsbeschreibung und Replikation
}

\section{Better Reporting of Interventions: Template for Intervention Description and Replication (TIDieR) Checklist and Guide}

\author{
Autoren \\ Die Autoren sind am Ende des Beitrags gelistet. \\ Institut \\ Die Institute sind am Ende des Beitrags gelistet.
}

\author{
Schlüsselwörter \\ - Leitlinien zur Forschungs- \\ berichterstattung \\ - Interventionsbeschreibung \\ - Replizierbarkeit
}

Key words

- reporting guidelines

- intervention description

- replicability
Bibliografie

DOI http://dx.doi.org/

10.1055/s-0041-111066

Online-Publikation: 29.1.2016

Gesundheitswesen 2016;

78: 175-188

(c) Georg Thieme Verlag KG

Stuttgart · New York

ISSN 0941-3790

Korrespondenzadresse

Dr. Sebastian Voigt-Radloff

Cochrane Germany

University Freiburg - Medical

Centre

Berliner Alle 29

79110 Freiburg

sebastian.voigt@uniklinik-

freiburg.de
Bearbeitete Übersetzung des Beitrags

Better reporting of interventions: tem-

plate for intervention description and

replication (TIDieR) checklist and guide

(DOI 10.1136/bmj.g1687) aus dem

Englischen:

\section{S. Voigt-Radloff \\ A. Blümle \\ J. Meerpohl}

Cochrane Deutschland, Universitätsklinikum

Freiburg, Freiburg

\section{Zusammenfassung}

$\nabla$

Nur wenn Interventionsbeschreibungen vollständig veröffentlicht sind, können Kliniker und Patienten Interventionen, die sich als nützlich erwiesen haben, verlässlich umsetzen und andere Forscher die Studienergebnisse replizieren oder darauf aufbauen. Die Qualität von Interventionsbeschreibungen in wissenschaftlichen Publikationen ist bemerkenswert gering. Um die Vollständigkeit der Berichterstattung und damit die Replizierbarkeit von Interventionen zu verbessern, entwickelte eine internationale Gruppe von Experten und Interessensvertretern die Checkliste zur Interventionsbeschreibung und Replikation (TIDieR). Der Prozess beinhaltete eine Literaturrecherche zu relevanten Checklisten und wissenschaftlichen Untersuchungen, eine Delphi-Umfrage mit internationalen Experten zur Steuerung der Item-Auswahl und eine Expertenkonferenz. Die daraus resultierende 12-Item-TIDieR Checkliste (Bezeichnung, Warum, Was (Materialien), Was (Verfahren), Wer intervenierte, Wie, Wo, Wann und Wieviel, Anpassungen, Modifikationen, Wie gut (geplante Durchführungskontrolle), Wie gut (tatsächliche Durchführung)) ist eine Erweiterung des CONSORT 2010 Statements (Item 5) und des SPIRIT

\section{Abstract}

$\nabla$

Without a complete published description of interventions, clinicians and patients cannot reliably implement interventions that are shown to be useful, and other researchers cannot replicate or build on research findings. The quality of description of interventions in publications, however, is remarkably poor. To improve the completeness of reporting, and ultimately the replicability, of interventions, an international group of experts and stakeholders developed the Template for Intervention Description and Replication (TIDieR) checklist and guide. The process involved a literature review for relevant checklists and research, a Delphi survey of an international panel of experts to guide item selection, and a face-to-face panel meeting. The resultant 12-item TIDieR checklist (brief name, why, what (materials), what (procedure), who intervened, how, where, when and how much, tailoring, modifications, how well (planned), how well (actually carried out)) is an extension of the CONSORT 2010 statement (item 5) and the SPIRIT 2013 statement (item 11). While the emphasis of the checklist is on trials, the guidance is intended to apply across all evaluative study designs. This paper presents the TIDieR checklist and guide, with a detailed explanation of each item, and examples of good reporting. The TIDieR checklist and guide should improve the reporting of interventions and make it easier for authors to structure the accounts of their interventions, reviewers and editors to assess the descriptions, and readers to use the information. 
2013 Statements (Item 11). Während der Fokus der Checkliste auf klinischen Studien liegt, kann die erweiterte Anleitung bei allen evaluativen Studiendesigns herangezogen werden. Dieser Artikel präsentiert die TIDieR Checkliste und Anleitung mit Erklärung und Erläuterung jedes einzelnen Items sowie Beispielen guter Berichterstattung. Die TIDieR Checkliste und Anleitung sollen das Berichten von Interventionen verbessern und Autoren eine Hilfe bieten, die Berichterstattung ihrer Interventionen zu strukturieren, Gutachtern und Herausgebern, die Beschreibungen zu beurteilen und Lesern, die Informationen zu nutzen.

\section{Einleitung}

Die Evaluation von Interventionen ist ein bedeutendes Forschungsfeld, dennoch ist die Qualität der Interventionsbeschreibungen in Publikationen noch immer bemerkenswert schlecht. Ohne eine vollständig publizierte Interventionsbeschreibung können andere Forscher die Studienergebnisse weder replizieren noch darauf aufbauen. Dadurch werden Kliniker, Patienten und andere Entscheidungsträger auch bei wirksamen Interventionen im Unklaren darüber gelassen, wie diese zuverlässig implementiert werden können. Eine Interventionsbeschreibung umfasst mehr als die Bezeichnung oder die reine Auflistung der Interventionsbestandteile. Alle Hauptmerkmale - einschließlich Dauer, Dosierung oder Intensität, Durchführungsmodus, Kernprozeduren und Monitoring - können die Wirksamkeit und Replizierbarkeit beeinflussen, werden aber oft gar nicht oder nur unzureichend beschrieben. Bei komplexen Interventionen sind diese Details für jede Komponente der Intervention erforderlich. So zeigte z.B. eine neuere Analyse, dass nur $11 \%$ von 262 Studien zu Chemotherapie bei Krebs die Details zur Studienbehandlung vollständig angaben [1]. Am häufigsten fehlten Angaben zu Dosisumstellungen und medikamentösen Vorbehandlungen. Bei $16 \%$ der Studien fehlten sogar Angaben zur Applikationsform. Nicht-pharmakologische Interventionen sind oft noch unvollständiger beschrieben: eine Analyse von Studien und Übersichtsarbeiten zeigte, dass $67 \%$ der medikamentösen, jedoch nur $29 \%$ der nicht-pharmakologischen Interventionen adäquat beschrieben waren [2]. Eine aktuelle Untersuchung zu 137 Interventionen aus 133 Studien fand, dass in Primärpublikationen, Referenzen, Anhängen oder angegeben Webseiten nur 39\% der nicht-medikamentösen Interventionen ausreichend beschrieben waren [3]. Dies verbesserte sich, wenn auch nur auf 59\%, wenn Autoren für zusätzliche Informationen kontaktiert wurden - ein Arbeitsschritt, für den nahezu kein Kliniker und nur wenige Forscher Zeit erübrigen können.

Im Item 5 empfiehlt das ,Consolidated Standards of Reporting Trials (CONSORT) 2010 Statement‘ [4], dass Autoren die Interventionen in jeder Gruppe mit präzisen Details berichten sollten, einschließlich wie und wann die Interventionen durchgeführt wurden, um eine Replikation der Studie zu ermöglichen. Für diese durchaus angemessene Empfehlung scheint jedoch weitere Anleitung notwendig. Denn obwohl viele Zeitschriften das CONSORT Statement unterstützen, ist die Berichterstattung von Interventionen defizitär. Das Problem besteht zum einen im mangelnden Bewusstsein der Autoren für die Inhalte guter Beschreibungen und zum anderen in der mangelnden Beachtung und Wertschätzung durch Gutachter und Herausgeber [5].

Einige der CONSORT Erweiterungen beinhalten weiterführende Anleitungen zur Beschreibung von Interventionen, so etwa die
CONSORT Erweiterung für nicht-pharmakologische Interventionen [6] und für spezifische Interventionen wie die CONSORT Erweiterung zu Akupunktur und Phytotherapie [7,8]. Die Anleitung für den Inhalt von Studienprotokollen SPIRIT (Standard Protocol Items: Recommendations for interventional Trials), gibt Empfehlungen für die Interventionsbeschreibung in Protokollen [9]. Jedoch ist eine allgemeingültige und umfassendere Anleitung erforderlich ebenso wie robuste Verfahren zur Implementation dieser Empfehlungen. Wir entwickelten eine Erweiterung von Item 5 des CONSORT 2010 Statements und Item 11 des SPIRIT 2013 Statements in Form einer Checkliste und Anleitung mit dem Titel TIDieR (Template for Intervention Description and Replication), um die Vollständigkeit der Berichterstattung und damit die Replizierbarkeit von Interventionen zu verbessern. Dieser Artikel beschreibt die Methoden der Checklistenentwicklung und Konsensbildung und präsentiert für jedes einzelne Item Erklärungen, Erläuterungen und Beispiele für gute Berichterstattung. Auch wenn der Fokus der Checkliste auf klinischen Studien liegt, kann die Anleitung bei allen evaluativen Studientypen wie klinischen Studien, Fall-Kontroll-Studien und Kohorten-Studien Anwendung finden.

\section{Methoden zur Entwicklung der TIDieR Checkliste und Anleitung \\ $\nabla$}

Die Entwicklung der Checkliste folgte dem vom EQUATOR Netzwerk vorgeschlagenen methodischen Konzept für die Entwicklung von Leitlinien für Forschungsberichte [10]. In Kooperation mit der CONSORT Gruppe gründeten wir ein TIDieR Steuerungskomitee (PPG, TCH, IB, RM, RP). Das Komitee erstellte eine Liste von 34 potentiellen Items basierend auf relevanten CONSORT Checklisten und Checklisten für die Berichterstattung disziplin-spezifischer oder anderer spezieller Arten von Interventionen. Außerdem überprüfte die Gruppe weitere Quellen mit Anleitungen zur Berichterstattung von Interventionen, welche durch eine umfassende Literaturrecherche identifiziert wurden, die durch eine anschließende Vorwärts-Rückwärts-Zitations-Suche ergänzt wurde.

Unter Einbezug eines breiten Spektrums an Expertise und Interessensvertretungen wendeten wir dann eine modifizierte Delphi-Konsensusmethodik [11] mit 2 Umfragerunden an. In der ersten Runde bewerteten die Umfrageteilnehmer die Einschlussfähigkeit jedes der vom Steuerungskomitee vorgeschlagenen 34 Items mit „ausschließen“, „möglich“ „wünschenswert“ oder „essentiell“. Einige Items aus der ersten Runde wurden umformuliert und zusammengeführt, danach wurden die priorisierten Items für die zweite Runde in 3 Gruppen aufgeteilt. Die erste Gruppe umfasste die 13 Items mit der höchsten Priorität (von $\geq 70 \%$ der Teilnehmer als „essentiell“ oder von $\geq 85 \%$ als „essentiell oder wünschenswert“ bewertet); die Teilnehmer wurden darauf hingewiesen, dass diese Items in die Checkliste eingeschlossen würden, wenn in der zweiten Runde keine starken Einwände gegen die Aufnahme eingebracht würden. Die zweite Gruppe beinhaltete 13 Items mit moderater Priorität („essentiell oder wünschenswert“ von $\geq 65 \%$ der Teilnehmer bewertet); die Teilnehmer wurden gebeten, die Einschlussfähigkeit dieser Items wiederum mit „ausschließen“, „möglich“, „wünschenswert“ oder „essentiell“ zu bewerten. Die dritte Gruppe umfasste 3 Items mit niedriger Priorität; die Teilnehmer wurden darauf hingewiesen, dass diese Items ausgeschlossen würden, wenn in der zweiten Runde keine starken Einwände gegen die 
Streichung eingebracht würden. Des Weiteren konnten die Teilnehmer in beiden Runden zusätzliche Items vorschlagen, Formulierungen kommentieren oder generelle Anmerkungen machen.

Die Delphi Teilnehmer $(n=125)$ waren Autoren wissenschaftlicher Artikel zur Interventionsbeschreibung, Kliniker, Autoren bereits existierender Leitlinien für Forschungsberichte, klinische Studienleiter, Methodiker oder Statistiker mit Expertise in klinischen Studien sowie Herausgeber von Zeitschriften. Sie wurden per E-Mail eingeladen, an beiden Runden der webbasierten Umfrage teilzunehmen. Die Antwortrate war $72 \%(n=90)$ für die erste Runde. Zur zweiten Runde wurden nur diejenigen eingeladen, die die erste Runde abgeschlossen und in eine weitere Teilnahme einwilligt hatten. Die Antwortrate für die zweite Runde lag bei $86 \%$ (74 von 86 eingeladenen Teilnehmern).

Nach den 2 Delphi-Runden wurden 13 Items in die vorläufige Checkliste aufgenommen und 13 als moderat eingestufte Items wurden zur weiteren Diskussion auf der Expertenkonferenz beibehalten. Die Ergebnisse der Delphi-Umfrage wurden während einer 2-tägigen Konsensus-Konferenz am 27.-28. März 2013 in Oxford, UK, vorgestellt. 13 eingeladene Experten aus verschiedenen Gesundheitsdisziplinen (siehe Autorenliste) und mit Expertise in der Entwicklung von Studien, methodischen Leitlinien oder Leitlinien für Forschungsberichte nahmen teil und sind alle Autoren dieses Artikels. Das Treffen begann mit einer Literaturübersicht zur Berichterstattung von Interventionen, gefolgt von der Präsentation des Delphi-Prozesses, des Entwurfs der 13-Item-Checkliste und der 13 zusätzlichen, als moderat eingestuften Items inklusive der Priorisierungsergebnisse und Kommentare. Die Konferenzteilnehmer diskutierten die vorgeschlagenen Items und einigten sich für jedes einzelne Item auf Einschluss und Formulierung.

Um sicherzustellen, dass die Checkliste die getroffenen Entscheidungen widerspiegelt, wurde sie nach der Konferenz an die Teilnehmer verteilt und der vorliegende Artikel mit Erklärungen und Erläuterungen entworfen. 26 Autoren von Interventionsstudien pilotierten den Artikelentwurf und die Erläuterungen mancher Items wurden präzisiert.

\section{Umfang der TIDieR Checkliste und Anleitung für die Beschreibung von Interventionen \\ $\nabla$}

Der wesentliche Zweck der TIDieR Checkliste ist es, Autoren zu einer so detaillierten Interventionsbeschreibung aufzufordern, dass eine Replikation möglich ist. Die Checkliste umfasst die Mindestanzahl an Items, die zur Interventionsbeschreibung empfohlen werden. Autoren sollten zusätzliche Informationen zur Verfügung stellen, wo sie es für die Replikation einer Intervention für notwendig erachten.

Die meisten TIDieR Items sind für die meisten Interventionen relevant und auch bei augenscheinlich einfachen medikamentösen Interventionen anzuwenden, die manchmal schlecht beschrieben sind [2]. Betrachten wir die Komponenten für die Evaluation einer Intervention nach dem PICO-Schema mit Population, Intervention, Comparison (Vergleichsbehandlung) und Outcome (Endpunkt), so stellt TIDieR die Handlungsempfehlung für die Berichterstattung der Intervention, Vergleichsbehandlung und - falls relevant - der Ko-Interventionen dar.

Andere Elemente wie Population, Endpunkte und methodische Merkmale sind für randomisierte Studien über die entsprechen- den Items der CONSORT 2010 oder SPIRIT 2013 Checklisten abgedeckt und für andere Studientypen über weitere Checklisten, wie das STROBE Statement [12]. Daher wurden sie in der TIDieR Checkliste nicht nochmals ausgeführt.

Die Reihenfolge der Items in der Checkliste stellt nicht zwingend die Reihenfolge dar, in der die Informationen präsentiert werden müssen. Es ist auch möglich, mehrere Items in einem Satz zu beschreiben. Z.B. können Informationen zu den eingesetzten Materialien (Punkt 3) und Prozessen (Punkt 4) zusammengefasst werden (Beispiel 3c).

Wir möchten betonen, dass unsere Definition von „Intervention“ sich auch auf die Beschreibung der Vergleichsinterventionen erstreckt. Kontroll- und Ko-Interventionen sind oft besonders schlecht beschrieben: „übliche Behandlung“ ist keine ausreichende Beschreibung. Beim Berichten kontrollierter Studien sollten Autoren soweit möglich ähnlich detailliert wie bei der Interventionsgruppe beschreiben, welche Behandlung die Teilnehmer der Kontrollgruppe erhielten. Umfassende Informationen zur Vergleichsbehandlung können helfen, die beobachtete Effektstärke zu erklären; scheinbar größere Effekte können gefunden werden, wenn die Behandlungsintensität in der Kontrollgruppe minimal ist [13]. Um die Behandlung in jeder Gruppe zu beschreiben, muss die Checkliste für jede Behandlungsgruppe wiederholt abgearbeitet werden.

Neben der Beschreibung der Interventionen oder Kontrollbedingungen in den verschiedenen Gruppen sollten die Autoren ebenso zugelassene Varianten der Interventionen erklären. Zur Einordnung ihrer Studien könnten Autoren das Kontinuum zwischen pragmatischen und streng experimentellen Untersuchungen heranziehen [14]. Erwarten die Autoren bspw. in einer pragmatischen Studie Varianten bei einzelnen Aspekten der Interventionen (wie etwa Unterschiede in der Routineversorgung der Kontrollgruppe in verschiedenen Studienzentren), dann sollten diese Varianten beim entsprechenden Item der Checkliste beschrieben werden.

Es ist uns bewusst, dass Format- und Längenbegrenzungen in der Druckversion von Zeitschriften verhindern, dass alle Informationen über die Interventionen in der Primärpublikation enthalten sind (die Primärpublikation berichtet die Hauptergebnisse der Interventionsevaluation). Zusätzlich zur Primärpublikation kann die von der TIDieR Checkliste geforderte Information daher an anderer Stelle berichtet werden, z. B. in Ergänzungsmaterialien online verlinkt mit dem Primärartikel, in veröffentlichten Studien-Protokollen, in anderen publizierten Artikeln oder auf Webseiten. Autoren sollten im Primärartikel genau angeben, wo zusätzliche Informationen zu finden sind (wie z.B. „Das Trainingsmanual ist im Online Appendix verfügbar“, „Verfügbar unter www....., oder „Details finden sich im publizierten Studien-Protokoll“). Werden weiterführende Informationen auf Internetseiten zur Verfügung gestellt, ist es erforderlich, dass die Webadresse über einen längeren Zeitraum unverändert bleibt.

\section{Die TIDieR Checkliste: Erklärungen und Erläuterungen} $\nabla$

Die Items der Checkliste sind in $\bullet$ Tab. 1 dargestellt. Eine englischsprachige Word-Version für Autoren und Gutachter zur direkten Bearbeitung ist auf der Internetseite des EQUATOR Netzwerkes zu finden (www.equator-network.org/reporting-guide lines/tidier/). Im Folgenden werden alle Items erklärt und Beispiele guter Berichterstattung aufgeführt. Die Literaturstellen zu den Beispielen sind in $\odot$ Tab. 2 zu finden. 
Item 1. Kurze Bezeichnung: Bezeichnung oder Umschreibung angeben, die die Intervention charakterisiert.

Beispiele

- 1a. Einzelne ... Dosis Dexamethason

- 1b. TREAD (TREAtment of Depression with physical activity) Studie

- 1c. Internetbasiertes, pflege-geleitetes Management-Programm von vaskulären Risikofaktoren zur Förderung des Selbstmanagements

\section{Erklärung}

Die präzise Bezeichnung oder Kurzbeschreibung einer Intervention ermöglicht die leichte Identifikation des Interventionstyps und erleichtert die Verknüpfung mit anderen Berichten zur selben Intervention. Intervention sind zu benennen (Beispiele 1a, 1b); jegliche Abkürzungen oder Akronyme zu erklären (Beispiel $1 b)$; oder Intervention kurz ohne ausführliche Erläuterungen zu umschreiben (ein oder 2 Zeilen, Beispiel 1c).

Item 2. Warum: Rationale, Theorien oder Ziele der für die Intervention wesentlichen Elemente beschreiben. Beispiele

- 2a. Dexamethason (10 mg) oder Plazebo wurden 15-20 min vor oder zusammen mit der ersten Antibiotikadosis verabreicht. ... Tierversuche haben gezeigt, dass eine durch Behandlung mit Antibiotika verursachte bakterielle Lyse zur Entzündung im Subarachnoidalraum führt, die zu einem ungünstigen Ergebnis beitragen kann [Referenzen]. Diese Studien zeigen ebenfalls, dass adjuvante Behandlungen mit Entzündungshemmern wie Dexamethason sowohl Entzündungen des Liquors als auch neurologische Residuen reduzieren [Referenzen].

- 2b. Selbstmanagement bei der Antikoagulantien-Therapie könnte zu einer individualisierteren Herangehensweise, zunehmender Patientenverantwortung und gesteigerter Compliance führen, was zu einer verbesserten Steuerung der Antikoagulation führen könnte.

- 2c. Das Interventionsprogramm zielte auf den Vorsatz und die Ausführung körperlicher Aktivitäten ab. Basierend auf der Theorie für geplantes Verhalten wurden als Mediatoren die instrumentelle und affektive Haltung, subjektive Normen und die wahrgenommene Verhaltenskontrolle hypothetisiert.

- 2d. Wir wählten einen Keil von $5^{\circ}$, da größere Keile durch den Träger schlechter toleriert werden [Referenz] und in einen normalen Schuh schlechter eingepasst werden können.

\section{Erklärung}

Die Einbeziehung der Rationale sowie von Theorien oder Zielen, die eine Intervention oder die Komponenten einer komplexen Intervention [15] untermauern, können anderen helfen zu unterscheiden, welche Elemente essentiell und welche optional oder nebensächlich sind. Z.B. ist die Farbe der Kapseln, die bei einer pharmakologischen Intervention verwendet werden, wahrscheinlich nicht essentiell für die Effektivität der Intervention und braucht deshalb nicht berichtet werden. Der in manchen Berichten verwendete Begriff „aktiver Wirkstoff“ bezieht sich auf diejenigen Komponenten einer Intervention, die spezifisch mit den Effekten einer Intervention in Verbindung gebracht werden können, nämlich in dem Sinne, dass die Intervention unwirksam wäre, wenn sie weggelassen würde [16]. Der be- kannte oder angenommene Wirkungsmechanismus der aktiven Bestandteile der Intervention sollte beschrieben werden.

Beispiel 2a legt die Rationale für die Behandlung der bakteriellen Meningitis mit Dexamethason zusätzlich zu einem Antibiotikum dar. Interventionen zur Verhaltensänderung oder Implementation erfordern eventuell andere Beschreibungsformen, die Grundprinzipien aber sind die gleichen.

Zusammen mit der Darstellung der Interventionskomponenten kann es ebenfalls sinnvoll sein, die theoretischen Grundlagen und angenommenen Wirkungsmechanismen der Intervention zu beschreiben (Beispiele 2b, 2c) [17-19]. Die Rationale für ein wichtiges Element einer Intervention kann manchmal pragmatisch bedingt sein und sich auf die Akzeptanz der Intervention durch die Teilnehmer beziehen (Beispiel 2d).

Item 3. Was (Materialien): Jegliches Informations- oder gegenständliches Material, das in der Intervention genutzt wurde, beschreiben, einschließlich Material, das an die Studienteilnehmer ausgegeben oder bei der Behandlung selbst oder beim Training der Behandelnden eingesetzt wird. Informieren, wie auf Material zugegriffen werden kann (z. B. OnlineAnhänge, Internetseiten).

Beispiele

- 3a. Das Ausbildungsmaterial beinhaltete ein 12-minütiges Cartoon... Die Präsentation des Cartoons wurde ergänzt durch Diskussionen im Klassenzimmer, die Ausstellung des gleichen Posters, das für die Kontrollgruppe verwendet wurde (siehe Schaubild in Anhang 4), das Verteilen einer Broschüre, die die Kernaussagen des Cartoons zusammenfasst, sowie durch Zeichen- und Schreibwettbewerbe, um die Botschaften zu verstärken ... Das Cartoon kann unter NEJM.org oder unter [bereitgestellte URL] abgerufen werden. Ein spezieller Trainings-Workshop für Lehrer wurde vor Studienbeginn durchgeführt (für Details siehe Protokoll, erhältlich unter NEJM.org).

- 3b. Die Interventionsgruppe erhielt ein Trainingsprogramm für die Beratung zu Verhaltensänderungen namens ,Talking Lifestyle learning programme', das niedergelassene Ärzte durch ein Portfolio-geleitetes Set von Lernaktivitäten führt. Einzelheiten zu Interventionsinhalten und Trainingsprogramm sind unter [URL, Login und Passwort bereit gestellt] zu finden... Kasten 1 bietet eine detailliertere Beschreibung der Komponenten des Trainingsprogramms.

- 3c. Die Gruppe vor Ort erhielt eine sonografisch gesteuerte Injektion von $2 \mathrm{~mL}(10 \mathrm{mg} / \mathrm{mL})$ Triamcinolon [Name und Hersteller des Handelspräparats] und $5 \mathrm{~mL}(10 \mathrm{mg} / \mathrm{mL})$ Lidocain-Hydrochlorid [Name und Hersteller des Handelspräparats] in die Bursa subacromialis und eine intramuskuläre Injektion von $4 \mathrm{~mL}(10 \mathrm{mg} / \mathrm{mL})$ Lidocain-Hydrochlorid in die obere Glutealregion.

\section{Erklärung}

Eine vollständige Interventionsbeschreibung sollte darstellen, welche unterschiedlichen Interventions- und Informationsmaterialien als Teil der Intervention verwendet wurden (dies umfasst normalerweise nicht die Einwilligungserklärungen zur Studienteilnahme, es sei denn, diese beinhalten schriftliche Instruktionen über die Intervention, die an keiner anderen Stelle bereitgestellt werden). Interventionsmaterialien sind die am häufigsten fehlenden Elemente von Interventionsbeschreibun- 
Tab. 1 Items der Checkliste zur Interventionsbeschreibung und Replikation (TIDieR): Notwendige Informationen für eine präzise Beschreibung von Interventionen.

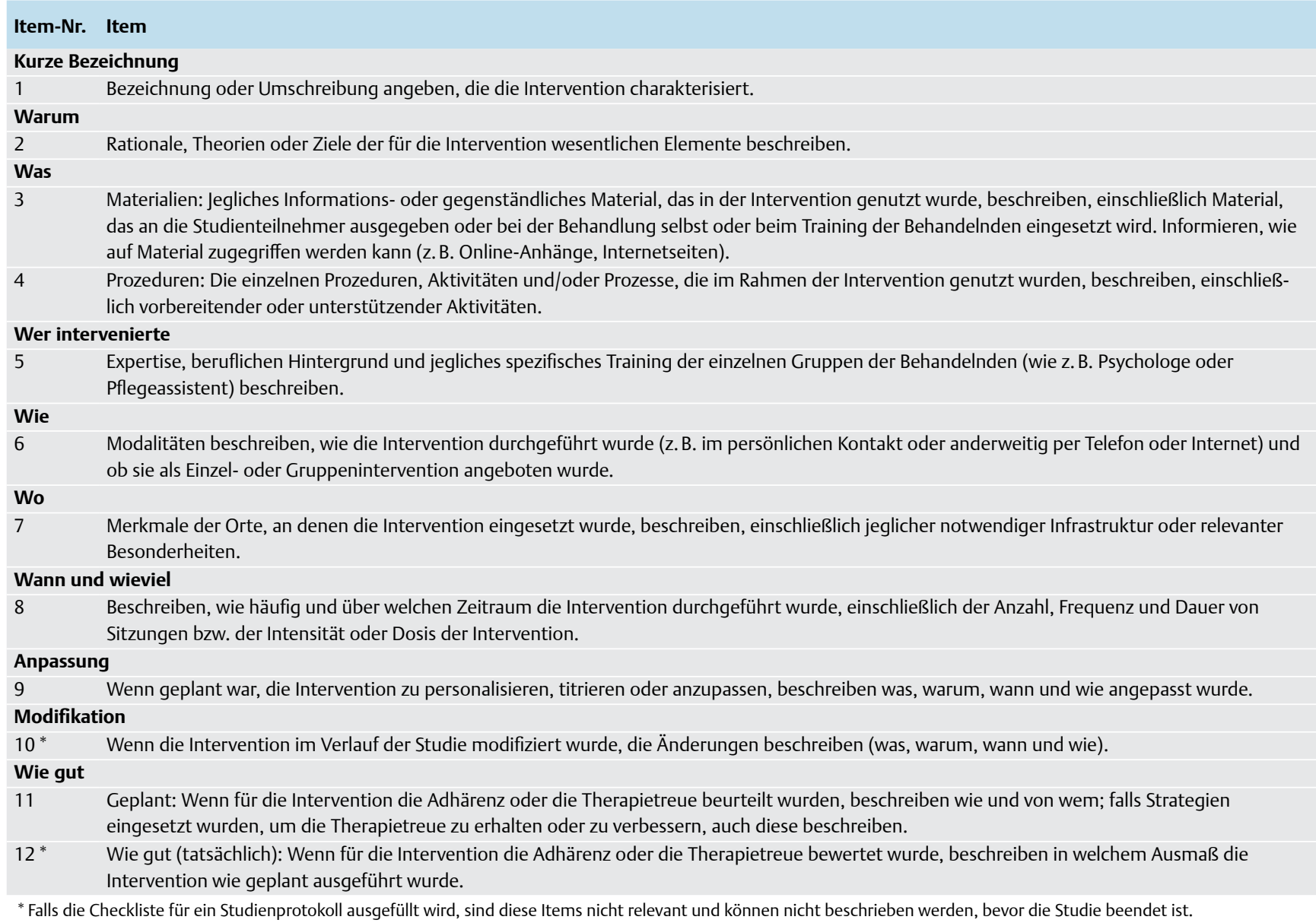

gen. [3] Die Liste von Materialien ist vergleichbar mit den „Zutaten“ für ein Rezept. Sie kann Materialien für die Studienteilnehmer (Beispiel 3a), Trainingsmaterialien für Behandelnde (Beispiele 3a, 3b) oder die eingesetzten chirurgischen Instrumente oder Medikamente einschließlich Hersteller (Beispiel 3c) beinhalten. Bei manchen Interventionen können Materialien und Prozeduren (Item 4) gemeinsam beschrieben werden (Beispiele 3c, 4c). Sind Informationen zu lang oder zu komplex für die Primärpublikation, sollten andere Optionen und Formate genutzt werden, um die Informationen zu Materialien bereit zu stellen. In der Primärpublikation sollte dann detailliert angegeben werden, wo diese Informationen zu finden sind (Beispiele 3a, 3b).

Item 4: Was (Prozeduren): Die einzelnen Prozeduren, Aktivitäten und/oder Prozesse, die im Rahmen der Intervention genutzt wurden, beschreiben, einschließlich vorbereitender oder unterstützender Aktivitäten.

Beispiele

- 4a. Die TREPP [transrectus sheath preperitoneal] Technik kann unter Spinalanästhesie durchgeführt werden. Um den preperitonealen Raum zu erreichen, wird eine $5 \mathrm{~cm}$ lange, gerade Inzision ca. $1 \mathrm{~cm}$ über dem Schambein durchgeführt. Die anteriore Rektusscheide wird eröffnet, ebenso die darunter liegende Fascia Transversalis [Bild]. Nach der Retraktion der mittleren Muskelfasern werden die inferioren epigastrischen Venen und Arterien identifiziert und ebenfalls mittig weggezogen.
- 4b. ... wurde eine geeignete Vene für die Kanülierung identifiziert. Gemäß der standardisierten Verfahrensanweisung wurde die darüber liegende Haut mit einem Alkoholtupfer abgewischt und trocknen gelassen. Der Studienleiter verabreichte das zugewiesene Spray aus einer Distanz von ca. $12 \mathrm{~cm}$ für 2 Sekunden. Diese Technik verhinderte die „Vereisung“" von Vapocoolant auf der Haut. Das flüssige Spray konnte auf der Haut bis zu 10 Sekunden verdunsten. Die Fläche wurde erneut mit einem Alkoholtupfer abgewischt; unmittelbar im Anschluss erfolgte die Kanülierung. Die Kanülierung musste innerhalb von 15 Sekunden nach Verabreichung des Sprays durchgeführt werden.

- 4c. ... 3 Übungsphasen von jeweils 5 min unter Aufsicht eines Physiotherapeuten. Die erste Phase bestand aus $2 \mathrm{~min}$ Indoor-Jogging, einer Minute Treppensteigen (3 Etagen) und 2 min Radfahren auf einem Ergometer. Der Widerstand des Ergometers wurde so eingestellt, dass eine erhöhte Atemfrequenz der Teilnehmer während der 2 min Radfahren sichergestellt war. Am Ende der ersten Phase wurde der Patient instruiert, einige tiefe und verlängerte Atemzüge mit beschleunigte Ausatmung bei geöffneter Glottis, die sogenannte forcierte Ausatemtechnik, durchzuführen mit abschließendem Husten und Sputumauswurf. Dieses Reinigungsmanöver wurde für 1,5 min durchgeführt. Die zweite Phase bestand aus einem 5-mal wiederholten einminütigen Dehnen, gefolgt von dem oben beschriebenen Ausatmungsmanöver. Die dritte Phase umfasste durchgehendes Trampolinspringen auf ei- 
Tab. 2 Liste der Referenzen, die in den Beispielen genutzt wurden

\section{Beispiele Referenzen}

Gallagher LTQ, Hill C, Keamy Jr DG, Williams M, Hansen M, Maurer R, et al. Perioperative dexamethasone administration and risk of bleeding following tonsillectomy in children: a randomized controlled trial. JAMA 2013; 308: 1221-1226

Chalder M, Wiles NJ, Campbell J, Hollinghurst SP, Haase AM, Taylor AH, et al. Facilitated physical activity as a treatment for depressed adults: randomised controlled trial. BM] 2012; 344: e2758

Vernooij JWP, Kaasjager HAH, van der Graaf Y, Wierdsma J, Grandjean HMH, Hovens MMC, et al. Internet based vascular risk factor management for patients with clinically manifest vascular disease: randomised controlled trial. BMJ 2012; 344: e3750

De Gans JD, van de Beek D. Dexamethasone in adults with bacterial meningitis. Engl J Med 2002; 347: 1549-1556

Cromheecke ME, Levi M, Colly LP, de Mol B], Prins MH, Hutten BA, et al. Oral anticoagulation self-management and management by a specialist anticoagulation clinic: a randomised cross-over comparison. Lancet 2000; 356: 97-102

Hardeman W, Kinmonth AL, Michie S, Sutton S. Impact of a physical activity intervention program on cognitive predictors of behaviour among adults at risk of type 2 diabetes (ProActive randomised controlled trial). Int J Behav Nutr Phys Act 2009; 6: 16

Bennell KL, Bowles K, Payne C, Cicuttini F, Williamson E, Forbes A, et al. Lateral wedge insoles for medial knee osteoarthritis: 12 month randomised controlled trial. BM] 2011; 342: d2912

Bieri F, Gray DJ, Williams GM, Raso G, Li Y-S, Yuan L, et al. Health-education package to prevent worm infections in Chinese schoolchildren. N Engl 」 Med 2013; 368: 1603-1612

Butler CC, Simpson SA, Hood K, Cohen D, Pickles T, Spanou C, et al. Training practitioners to deliver opportunistic multiple behaviour change counselling in primary care: a cluster randomised trial. BM] 2013; 346: f1191

Ekeberg OM, Bautz-holter E, Tveita EK, Juel NG, Kvalheim S. Subacromial ultrasound guided or systemic steroid injection for rotator cuff disease: randomised double blind study. BM] 2009; 338: a3112

Koning G, Andeweg C, Keus F, van Tilburg M, van Laarhoven C, Akkersdijk W. The transrectus sheath preperitoneal mesh repair for inguinal hernia: technique, rationale, and results of the first 50 cases. Hernia 2012; 16: 295-299

Hijazi R, Taylor D, Richardson J. Effekt of topical alkane vapocoolant spray on pain with intravenous cannulation in patients in emergency departments: randomised double blind placebo controlled trial. BMJ 2009; 338: b215

Reix P, Aubert F, Werck-Gallois M-C, Toutain A, Mazzocchi C, Moreux N, et al. Exercise with incorporated expiratory manoeuvres was as effective as breathing techniques for airway clearance in children with cystic fibrosis: a randomised crossover trial. J Physiother 2012; 58: 241-247

Zurovac D, Sudoi RK, Akhwale WS, Ndiritu M, Hamer DH, Rowe AK, et al. The effekt of mobile phone text-message reminders on Kenyan health workers' adherence to malaria treatment guidelines: a cluster randomised trial. Lancet 2011; 378: 795-803

Huang SS, Septimus E, Kleinman K, Moody J, Hickok J, Avery TR, et al. Targeted versus universal decolonisation to prevent ICU infection. N Engl J Med 2013; 368: 2255-2265

Doherty T, Tabana H, Jackson D, Swanevelder S, Fox MP, Thorson A. Effekt of home based HIV counselling and testing intervention in rural South Africa: cluster randomised trial. BMJ 2013; 3481: 1-11

Tiernan J, Hind D, Watson A, Wailoo AJ, Bradburn M, Shephard N, et al. The HubBLe trial: haemorrhoidal artery ligation (HAL) versus rubber band ligation (RBL) for haemorrhoids. BMC Gastroenterol 2012; 12: 153

Wiles N, Thomas L, Abel A, Ridgway N, Turner N, Campbell J, et al. Cognitive behavioural therapy as an adjunct to pharmacotherapy for primary care based patients with treatment resistant depression: results of the CoBalT randomised controlled trial. Lancet 2013; 381: 375-384

Kaner E, Bland M, Cassidy P, Coulton S, Dale V, Deluca P, et al. Effectiveness of screening and brief alcohol intervention in primary care (SIPS trial): pragmatic cluster randomised controlled trial. BMJ 2013; 346: e8501

Thomas S, Thomas PW, Kersten P, Jones R, Green C, Nock A, et al. A pragmatic parallel arm multi-centre randomised controlled trial to assess the effectiveness and cost-effectiveness of a group-based fatigue management programme (FACETS) for people with multiple sclerosis. J Neurol Neurosurg Psychiatry 2013; 84: 1092-1099

Bojang K, Akor F, Conteh L, Webb E, Bittaye O, Conway DJ, et al. Two strategies for the delivery of IPTc in an area of seasonal malaria transmission in the Gambia: a randomised controlled trial. PLoS Med 2011; 8: e1000409

Ybarra M, Bağcı Bosi A., Korchmaros J, Emri S. A text messaging-based smoking cessation program for adult smokers: randomised controlled trial. J Med Internet Res 2012; 14: e172

Kessler D, Lewis G, Kaur S, Wiles N, King M, Weich S, et al. Therapist-delivered internet psychotherapy for depression in primary care: a randomised controlled trial. Lancet 2009; 374: 628-634

Chumbler NR, Quigley P, Li X, Morey M, Rose D, Sanford J, et al. Effects of telerehabilitation on physical function and disability for stroke patients: a randomised, controlled trial. Stroke 2012; 43: 2168-2174

Halterman J, Szilagyi P, Fisher S, Fagnano M, Tremblay P, Conn K, et al. Randomised controlled trial to improve care for urban children with asthma: results of the school-based asthma therapy trial. Arch Pediatr Adolesc Med 2013; 165: 262-268

Van den Broek NR, White SA, Goodall M, Ntonya C, Kayira E, Kafulafula G, et al. The APPLe study: a randomised, community-based, placebo-controlled trial of azithromycin for the prevention of preterm birth, with meta-analysis. PLoS Med 2009; 6: e1000191

Cartwright M, Hirani SP, Rixon L, Beynon M, Doll H, Bower P, et al. Effekt of telehealth on quality of life and psychological outcomes over 12 months (Whole Systems Demonstrator telehealth questionnaire study): nested study of patient reported outcomes in a pragmatic, cluster randomised controlled trial. BMJ 2013; 346: f653

Lewycka S, Mwansambo C, Rosato M, Kazembe P, Phiri T, Mganga A, et al. Effekt of women's groups and volunteer peer counselling on rates of mortality, morbidity, and health behaviours in mothers and children in rural Malawi (MaiMwana): a factorial, cluster-randomised controlled trial. Lancet 2013; 381: 1721-1735

Murphy AW, Cupples ME, Smith SM, Byrne M, Byrne MC, Newell J. Effekt of tailored practice and patient care plans on secondary prevention of heart disease in general practice: cluster randomised controlled trial. BM] 2009; 339: b4220

Shakur H, Roberts I, Bautista R, Caballero J, Coats T, Dewan Y, et al. Effects of tranexamic acid on death, vascular occlusive events, and blood transfusion in trauma patients with significant haemorrhage (CRASH-2): a randomised, placebo-controlled trial. Lancet 2010; 376: 23-32 Free C, Knight R, Robertson S, Whittaker R, Edwards P, Zhou W, et al. Smoking cessation support delivered via mobile phone text messaging (txt2stop): a single-blind, randomised trial. Lancet 2011; 378: 49-55 
Tab. 2 Fortsetzung.

\section{Beispiele Referenzen}

8c McDermott MM, Ades P, Guralnik JM, Nelson M, Horn L Van, Garside D, et al. Treadmill exercise and resistance training in patients with peripheral arterial disease with and without intermittent claudication. JAMA 2009; 301: 165-174

8d Morrell CJ, Slade P, Warner R, Paley G, Dixon S, Walters SJ, et al. Clinical effectiveness of health visitor training in psychologically informed approaches for depression in postnatal women: pragmatic cluster randomised trial in primary care. BM] 2009; 338: a3045

9a Stewart S, Carrington MJ, Swemmer CH, Anderson C, Kurstjens NP, Amerena J, et al. Effekt of intensive structured care on individual blood pressure targets in primary care: multicentre randomised controlled trial. BMJ 2012; 345: e7156

9b Lee W-J, Wang W, Lee Y-C, Huang M-T, Ser K-H, Chen J-C. Laparoscopic mini-gastric bypass: experience with tailored bypass limb according to body weight. Obes Surg 2008; 18: 294-299

9c McDermott MM, Ades P, Guralnik JM, Nelson M, Horn L Van, Garside D, et al. Treadmill exercise and resistance training in patients with peripheral arterial disease with and without intermittent claudication. JAMA 2009; 301: 165-174

9d Davidson KW, Rieckmann N, Clemow L, Schwartz JE, Shimbo D. Enhanced depression care for patients with acute coronary syndrome and persistent depressive symptoms. JAMA Intern Med 2010; 170: 600-608

10a Kaner E, Bland M, Cassidy P, Coulton S, Dale V, Deluca P, et al. Effectiveness of screening and brief alcohol intervention in primary care (SIPS trial): pragmatic cluster randomised controlled trial. BMJ 2013; 346: e8501

10b Wake M, Lycett K, Clifford SA, Sabin MA, Gunn J, Gibbons K, et al. Shared care obesity management in 3-10 year old children: 12 month outcomes of HopSCOTCH randomised trial. BM] 2013; 346: $\mathrm{f3092}$

11a Kapiteijn E, Marijnen C, Nagtegaal I, Putter H, Steup W, Wiggers T, et al. Preoperative radiotherapy combined with total mesorectal excision for resectable rectal cancer. N Engl J Med 2001; 345: 638-646

11b Morrison AP, French P, Stewart SLK, Birchwood M, Fowler D, Gumley AI, et al. Early detection and intervention evaluation for people at risk of psychosis: multisite randomised controlled trial. BM] 2012; 344: e2233

11c Thomas KS, Crook AM, Nunn 11c AJ, Foster K, Mason JM, Chalmers JR, et al. Penicillin to prevent recurrent leg cellulitis. N Engl J Med 2013; 368: 1695-1703

11d Murphy AW, Cupples ME, Smith SM, Byrne M, Leathem C, Byrne MC. The SPHERE Study. Secondary prevention of heart disease in general practice: protocol of a randomised controlled trial of tailored practice and patient care plans with parallel qualitative, economic and policy analyses. Trials 2005; 16: 1-16

12a Coombes BK, Bisset L, Brooks P, Khan A, Vincenzio B. Effekt of corticosteroid injection, physiotherapy, or both on clinical outcomes in patients with unilateral lateral epicondylalgia: a randomized controlled trial. JAMA 2013; 309: 461-9.

12b Dodd MJ, Cho MH, Miaskowski C, Krasnoff J, Bank KA. A randomised controlled trial of home-based exercise for cancer-related fatigue in women during and after chemotherapy with or without radiation therapy. Cancer Nurs 2010; 33: 245-257

12c Thomas KS, Crook AM, Nunn AJ, Foster K, Mason JM, Chalmers JR, et al. Penicillin to prevent recurrent leg cellulitis. N Engl J Med 2013; 368 : 1695-703. Kessler D, Lewis G, Kaur S, Wiles N, King M, Weich S, et al. Therapist-delivered internet psychotherapy for depression in primary care: a randomized controlled trial. Lancet 2009; 374: 628-634

12d Kessler D, Lewis G, Kaur S, Wiles N, King M, Weich S, et al. Therapist-delivered internet psychotherapy for depression in primary care: a randomized controlled trial. Lancet 2009; 374: 628-634

nem kleinen Trampolin. Es beinhaltete 2 min Springen, 2 min Springen mit Ballwerfen und -fangen und eine Minute Springen mit Weggeschlagen eines zugeworfenen Balls. Es folgte wiederum das Ausatmungsmanöver für 1,5 min. Nach der gesamten Maßnahme folgten 40 min Ruhe.

- 4d. Das gesamte Gesundheitspersonal, das die ambulante Konsultation in der Interventionsgruppe durchführte, erhielt über 6 Monate Mitteilungen per SMS zum Management bei Malaria. Die Kernaussagen bezogen sich auf Empfehlungen der nationalen, kenianischen Malaria-Leitlinien und Übungsmanualen [Referenzen].

- 4e. Vor-Ort-Aktivitäten wurden durch das Krankenhauspersonal implementiert, das für Initiativen zur Qualitätsverbesserung verantwortlich ist. Etablierte Kommunikationswege wurden genutzt, einschließlich gruppenspezifischer, computerbasierter Trainingsmodule und täglicher elektronischer Dokumentation durch Pflegepersonal für alle Gruppen. Krankenhäuser, die der Durchführung der Entkolonisierung zugewiesen wurden, erhielten vor Ort ein Training für das Bad in chlorhexidin-imprägnierter Kleidung... Pflegedirektoren führten mindestens 3 vierteljährliche Überprüfungen des Bades durch, einschließlich der Befragung von Mitarbeitern zu Protokolldetails. Zum Coaching führten die Untersucher mindestens monatlich gruppenspezifische Telefonkonferenzen durch, um die Umsetzung, Therapiebefolgung und jegliche neuen potenziell konfliktträchtigen Initiativen zu diskutieren.

\section{Erklärung}

Prozesse, Aktivitäten und Prozeduren, die die Behandelnden ausführen, sind zu beschreiben. Die obenstehende Rezept-Metapher weiterführend, bezieht sich dieses Item auf den ,Methoden'-Teil eines Rezepts und beschreibt, wo die Interventionsmaterialien („Zutaten“) eingebracht werden und was mit ihnen zu tun ist. Der Begriff „Prozedur“ kann sich auf die Abfolge der Schritte beziehen (Beispiele 3c, 4b) und wird in einigen Disziplinen explizit genutzt. In der Chirurgie beinhaltet er bspw. die präoperative Untersuchung und Optimierung, die Anästhesieart, die perioperative und postoperative Versorgung und Details der konkret eingesetzten chirurgischen Vorgehensweise (Beispiel 4a).

Beispiele für Prozesse oder Aktivitäten umfassen Zuweisungen, Screening, Indikationsstellung, Bewertung, Beratung, Behandlungseinheiten (Beispiel 4c), Telefonkontakte (Beispiel 4d) u.a.. Manche Interventionen, insbesondere komplexere, können zusätzliche Aktivitäten erforderlich machen, die die Intervention vorbereiten oder unterstützen. In manchen Disziplinen sind diese als Implementierungsaktivitäten bekannt und sollten ebenso beschrieben werden (Beispiel 4e). Erläuterungen zur Beschreibung von Prozeduren, die nicht für alle Patienten gleich sind, finden sich bei Item 9 (Anpassung).

Item 5. Wer intervenierte: Expertise, beruflichen Hintergrund und jegliches spezifisches Training 
der einzelnen Gruppen der Behandelnden (wie z. B. Psychologe oder Pflegeassistent) beschreiben Beispiele

- 5a. Nach Rücksprache mit den Dorfvorstehern wurden nur weibliche Berater in dieser ländlichen Region eingeschlossen, da es kulturell als unangemessen erachtet wurde, dass Männer Frauen ohne Anwesenheit des Ehemannes beraten... Die Auswahlkriterien für die Laien-Berater beinhalteten eine 12-jährige Schulzeit, einen Wohnsitz im Interventionsgebiet und Vorerfahrung in der Gemeinwesenarbeit.

- 5b. Die Prozedur ist einfach, basiert auf existierende chirurgische Fertigkeiten und hat eine kurze Lernkurve, wobei die Hersteller vor der selbständigen Durchführung mindestens 5 supervidierte Fälle empfehlen. Alle in die Studie involvierten Chirurgen werden dieses Training abgeschlossen und mehr als 5 Prozeduren vor der Rekrutierung in die Studie ausgeführt haben.

- 5c. Die Therapeuten erhielten mindestens einen Tag lang ein für die Studie spezifisches Training durch einen erfahrenen Therapeuten und Trainer in kognitiver Verhaltenstherapie (KVT) und in jedem Zentrum wöchentliche Supervision durch kompetente KVT-Supervisoren. Die Intervention wurde durch 11 Teilzeittherapeuten an 3 für den psychologischen Dienst des NHS repräsentativen Standorten ausgeführt [Referenz]. 10 der 11 Therapeuten waren weiblich, das Durchschnittsalter lag bei 39,2 Jahren (SD 8,1), und sie hatten durchschnittlich 9,7 Jahre (SD 8,1) als Therapeuten gearbeitet... 9 der 11 Therapeuten führten $97 \%$ der Interventionen durch mit einer Anzahl von 13 (6\%) bis 41 (18\%) Patienten pro Therapeut.

- 5d. ...die Kurzberatung zum Lebensstil wurde mit geschulten Schauspielern trainiert und auf Tonband aufgenommen. Die Beratungskompetenz wurde mithilfe des ,Behaviour Change Counselling Index' [Referenz] überprüft. Nur Praktiker, die einen geforderten Standard erreichten (ermittelt durch einen Konsens zwischen 3 unabhängigen klinischen Gutachtern), wurden zur Kurzberatung zum Lebensstil für die Studie zugelassen.

\section{Erklärung}

Der Begriff „Interventionsdurchführende“ bezieht sich auf Personen, die in Vorbereitung und Durchführung der Intervention involviert waren (sie z.B. mit den Klienten durchführten oder spezielle sonstige Aufgaben übernahmen). Dies ist besonders in Situationen wichtig, in denen die Expertise der Person und andere Besonderheiten (Beispiel 5a) die Ergebnisse der Intervention beeinflussen können. Wichtige, in der Beschreibung anzusprechende Themen wären: (a) Anzahl der involvierten Personen; (b) ihr beruflicher Hintergrund (z. B. Pflegekräfte, Ergotherapeuten, Chirurgen, Patienten als Experten); (c) die erforderliche Vorerfahrung, Expertise oder spezifischen Fertigkeiten und wie diese nachgeprüft wurden; (d) Details zu jedem zusätzlichen, interventionsspezifischen Training, das mit den Anbietern im Vorfeld (Beispiel 3b) oder während der Studie (Beispiel 5c) durchgeführt wurde; und die Frage, ob Kompetenzen zur Interventionsdurchführung vorab beurteilt (Beispiel $5 \mathrm{~d}$ ) oder während der Studie durchgehend überwacht wurden und ob Anbieter mit Kompetenzmängeln ausgeschlossen (Beispiel 5d) oder erneut trainiert wurden.

Weitere Informationen zu den Durchführenden können beinhalten, ob sie die Intervention als Teil ihrer normalen Funktion ausführen (Beispiel 3b), sie extra für die Studie rekrutiert wurden
(Beispiel 5c); ihre Zeit vergütet bekommen oder andere Anreize für die Interventionsdurchführung geboten wurden (wenn ja, welche) und ob vergüteter Zeiteinsatz oder andere Anreize notwendig sein könnten, um die Intervention zu replizieren.

Item 6. Wie: Modalitäten beschreiben, wie die Intervention durchgeführt wurde (z. B. im persönlichen Kontakt oder anderweitig per Telefon oder Internet) und ob sie als Einzel- oder Gruppenintervention angeboten wurde.

Beispiele

- 6a. Sitzungen ... wurden wöchentlich in Gruppen von 6-12 Personen abgehalten und moderiert durch...

- 6b. Arzneimittel wurden verabreicht durch ... Mitglieder des mobilen Teams für Reproduktions- und Kindesgesundheit... Teams besuchten jedes der Studiendörfer.

- 6c. Die Textnachrichten-Intervention „SMS-Turkey“ sendete 6 Wochen lang täglich Nachrichten, um den Teilnehmern Kenntnisse zu vermitteln, die ihnen helfen, mit dem Rauchen aufzuhören. Die Nachrichten wurden automatisch versendet, außer 2 und 7 Tage nach dem ersten Tag des Nichtrauchens.

- $6 \mathrm{~d}$.... vereinbarten ihre eigenen Termine online ... Teilnehmer und Therapeuten gaben Freitext direkt in den Computer ein und versendeten die Nachricht unmittelbar; es wurden keine anderen Medien oder Kommunikationsmittel benutzt.

- 6e. ... 3 einstündige Hausbesuche (Televisiten) durch einen trainierten Assistenten ... die Teilnehmer nutzten täglich ein Nachrichtengerät in ihrer Wohnung, das wöchentlich durch den Tele-Therapeuten überprüft wurde; und 5 telefonische Interventionseinheiten zwischen Tele-Therapeut und Teilnehmer.

\section{Erklärung}

Es ist zu spezifizieren, ob die Intervention jeweils mit einer Person (wie eine chirurgische Intervention) oder mit einer Patientengruppe durchgeführt wurde und die Gruppengröße ist anzugeben (Beispiel 6a). Es muss beschrieben werden, ob die Intervention im persönlichen Kontakt (Beispiel 6a) oder indirekt (über Telefon, Postweg, E-Mail, Internet, DVD, Kampagnen in Massenmedien, o.ä.) (Beispiele 6c und 6d), oder kombiniert (Beispiel 6e) durchgeführt wurde.

Wenn relevant, ist zu beschreiben, wer die Kontakte initiiert hat (Beispiel 6c) und ob die Sitzungen interaktiv waren (Beispiel 6d) oder nicht (Beispiel 6c). Jede weitere Besonderheit der Durchführung, die als wesentlich oder möglicherweise Ergebnis beeinflussend erachtet wird, ist zu beschreiben.

Item 7. Wo: Merkmale der Orte, an denen die Intervention eingesetzt wurde, beschreiben, einschließlich jeglicher notwendiger Infrastruktur oder relevanter Besonderheiten.

Beispiele

- 7a.... Medikation ... und wenn erforderlich ein Dosierer wurden der Schulkrankenpflegekraft zur Therapie unter direkter Beobachtung überlassen an den Tagen, an denen das Kind die Schule besuchte. Für Wochenenden und schulfreie Tage wurde ein zusätzliches Behältnis mit präventiver Medikation im Elternhaus deponiert. Die Pflegekraft des Kindes erhielt eine Einweisung in geeignete Techniken zur Verabreichung.

- 7b. Frauen wurden aus einer stadtnahen und 3 ländlichen Geburtskliniken in Süd-Malawi rekrutiert ... Tabletten wurden in der Klinik unter Aufsicht eingenommen. 
- 7c. ... Teilnehmer für die ... telemedizinische Studie waren über 3 soziodemografisch unterschiedliche Regionen in England verteilt (ländliches Cornwall, ländliches und städtisches Kent und städtisches Newham in London), die 4 staatliche Organisationen der Primärversorgung umfassen. Teilnehmer der Kontrollintervention hatten während des Studienzeitraums keine Geräte zur telemedizinischen Betreuung in ihrer Wohnung installiert. Die Kombination aus Hausnotruf und zentral überwachtem Rauchmelder war nicht ausreichend, um im Sinne der Studie als telemedizinische Betreuung klassifiziert zu werden.

- 7d. Die meisten Geburten in afrikanischen Ländern erfolgen zu Hause, vor allem in ländlichen Gegenden ... Sie identifizierten schwangere Frauen und führten 5 Hausbesuche während und nach der Schwangerschaft durch ... Die „Peer-Beraterinnen“ lebten in derselben Gemeinde, sodass die Vereinbarung der Besuche häufig informell erfolgte ... Beraterinnen erhielten... ein Fahrrad und ein T-Shirt.

- 7e. Dieses Dokument enthält einen Kasten mit dem Titel "Hauptmerkmale des Gesundheitssystems in Nordirland und Irland“, das relevante Aspekte zu Hausarztpraxen wie z. B. Finanzierung, Registrierung und Zugang zu freien Rezepten zusammenfasst.

\section{Erklärung}

In manchen Studien können die Interventionen an dem Ort durchgeführt werden, an dem die Teilnehmer auch rekrutiert oder die Daten gesammelt werden. Dann können Details zum Einsatzort bereits als Teil der Primärpublikation vorgesehen sein (wie z.B. in Item 4b des CONSORT 2010 Statement für Studienberichte gefordert). Falls hingegen die Intervention an anderen Orten durchgeführt, sollte dies genauer beschrieben werden. Im einfachsten Fall könnte der Ort z.B. die Wohnung der Teilnehmern zu Hause (Beispiel 7a), eine Altenpflegeeinrichtung, eine Schule (Beispiel 7a), eine klinische Ambulanz (Beispiel 7b), ein Krankenhauszimmer oder eine Kombination von Einsatzorten (Beispiel 7a) sein. Besondere Merkmale oder Gegebenheiten der Einsatzorte können für die Durchführung der Intervention relevant sein und sollten beschrieben werden (Beispiel 7e). Z. B. können sie sich auf das Land (Beispiel 7b), den Typ des Krankenhauses bzw. die Primärversorgung (Beispiel 7c), eine öffentlich oder privat finanzierte Versorgungseinrichtung, das Aktivitätsvolumen, Details des Gesundheitssystems oder die Verfügbarkeit bestimmter Einrichtungen oder Geräte (Beispiele 7c, 7d, 7e) beziehen. Diese Besonderheiten können verschiedene Aspekte der Intervention beeinflussen, wie etwa ihre Machbarkeit (Beispiel 7d) oder die Adhärenz der Behandelnden oder der Behandelten, und sind wichtig für diejenigen, die erwägen die Intervention zu replizieren.

Item 8. Wann und wie viel: Beschreiben, wie häufig und über welchen Zeitraum die Intervention durchgeführt wurde, einschließlich der Anzahl, Frequenz und Dauer von Sitzungen bzw. der Intensität oder Dosis der Intervention.

Beispiele

- 8a. ... eine Sättigungsdosis von $1 \mathrm{~g}$ Tranexam-Säure wurde über $10 \mathrm{~min}$ infundiert, gefolgt von einer intravenösen Infusion von $1 \mathrm{~g}$ über 8 Stunden.

- 8b. Die ersten 5 Wochen erhielten sie 5 Textnachrichten am Tag und danach wöchentlich 3 für die nächsten 26 Wochen.
- 8c. ... 3-mal wöchentlich Übungen für 24 Wochen ... Die Teilnehmer begannen mit 15 min Übung und steigerten sich auf $40 \mathrm{~min}$ in Woche 8. ... Zwischen Woche 8 und 24 wurde mindestens einmal pro Woche versucht, die Übungsintensität durch eine erhöhte Laufbandsteigung oder -geschwindigkeit zu steigern. Teilnehmer mit Beinbeschwerden wurden ermutigt, bis nahe an die Beschwerdegrenze zu trainieren. Teilnehmer ohne Beschwerden wurden angewiesen, bis zu einem Level von 12 bis 14 auf der Borg Skala zur subjektiv wahrgenommenen Anstrengung zu trainieren... [Referenz].

- 8d. ... führten für bis zu 8 Wochen bei den Frauen zu Hause wöchentlich einstündige Sitzungen durch ... beginnend etwa 8 Wochen nach der Entbindung.

\section{Erklärung}

Die erforderliche Information zu dem „wann und wie viel“ einer Intervention wird sich je nach Interventionsart unterscheiden. Für manche Interventionen sind bestimmte Aspekte wichtiger als andere. Z. B. sind für pharmakologische Interventionen meist die Dosis und der Applikationsplan wichtig (Beispiel 8a); für viele nicht-pharmakologische Interventionen wird das „wie viel“ jedoch durch die Dauer und Anzahl der Sitzungen beschrieben (Beispiele 8b, 8c). Bei Interventionen mit mehreren Sitzungen ist ein Ablaufplan erforderlich (Beispiel 8b), ebenso die Information, ob Anzahl, Terminierung und Intensität der Sitzungen festgelegt wurden (Beispiele 8b, 4c, 6a) und ob nach bestimmten Regeln variiert werden durfte und wie diese Regeln lauteten (Beispiel 8c). Die Anpassung der Intervention für einzelne Personen oder Gruppen wird in Item 9 (Anpassung) näher ausgeführt. Details über die zeitliche Terminierung der Intervention in Verhältnis zu relevanten Ereignissen können ein wichtiger Bestandteil der „wann“-Information sein (z.B. wie lange nach der Diagnose, den ersten Symptomen oder einem kritischem Ereignis begann die Intervention) (Beispiel 8d).

Wie unten in Item 12 beschrieben, kann die „Menge“ oder Dosis der Intervention, die die Teilnehmer tatsächlich erhielten, von der vorgesehenen Menge abweichen. Dieses Detail sollte beschrieben werden, üblicherweise im Ergebnisteil (Beispiele 12a-c).

Item 9. Anpassung: Wenn geplant war, die Intervention zu personalisieren, titrieren oder anzupassen, beschreiben was, warum, wann und wie angepasst wurde.

Beispiele

- 9a. Jene, die dem Interventionsarm zugewiesen wurden, befolgten ein intensives, gestuftes Managementprogramm mit Pflichtbesuchen bei ihrem Arzt in Woche 6, 10, 14 und 18 nach der Randomisierung zur Überprüfung des Blutdruck und ggf. Anpassung der Behandlung nach vordefinierten Algorithmen (s. Anhänge).

- 9b. Alle Patienten erhielten eine laparoskopische mini-gastrische Bypass-Operation ... das Bypass-Implantat wurde in Abhängigkeit vom präoperativen BMI des Patienten angepasst. Anstatt des Einsatzes eines einheitlichen Implantats bei allen Patienten wurde ein $150 \mathrm{~cm}$ Implantat bei einem BMI von 35 verwendet, und das Bypass-Implantat mit jeder nächst höheren BMI-Kategorie um $10 \mathrm{~cm}$ verlängert.

- 9c. Die Teilnehmer begannen die Übung bei $50 \%$ ihres $1 \mathrm{rm}$ [Repetitionsmaximum]. Über die ersten 5 Wochen wurden die Gewichte erhöht bis die Teilnehmer $80 \%$ ihres 1rm anhoben. Die Gewichte wurden monatlich nach Erreichen des $1 \mathrm{rm}$ angepasst oder nach Bedarf, um eine Trainingsintensität zu 
erreichen, die auf der Skala der subjektiv wahrgenommenen Anstrengung zwischen 12 und 14 lag.

- 9d. Entscheidungen zur stufenweisen Versorgung der Patienten ... wurden je nach Beantwortung des 9-Item-Patientengesundheitsfragebogens [Referenz] gefällt, der bei jedem Behandlungsbesuch angewendet und in achtwöchigen Intervallen formal ausgewertet wurde. Patienten, die keine a priori definierte Verbesserung erreichten, konnten nach Empfehlung des Behandlungsteams wählen, entweder zur alternativen Behandlung zu wechseln (z. B. von der Problemlösetherapie zur medikamentösen Therapie), die alternative Behandlung zusätzlich durchzuführen oder die ursprüngliche Behandlung zu intensivieren (für Details, siehe [Referenz]).

\section{Erklärung}

Individuell angepasste Interventionen sind nicht für alle Teilnehmer identisch. Es gibt mehrere Gründe, warum Interventionen individuell angepasst werden können. $\mathrm{Zu}$ diesen Gründen zählen z.B. das Titrieren, um die richtige „Dosis“ zu finden (Beispiel 9a), oder Präferenzen, Fähigkeiten oder die Situation der Teilnehmer (Beispiel 9b). Die Anpassung kann auch wichtiger Bestandteil der Intervention sein, wie etwa die zunehmende Intensität körperlichen Trainings (Beispiel 9c). Daher sollte eine kurze Begründung und Anleitung für die Anpassung zur Verfügung stehen. Diese Anleitung sollte auch alle Variablen/Konstrukte beschreiben, die für Beurteilung der Teilnehmer und daran anschließende Anpassung benötigt werden. Die Anpassung kann zu mehreren Zeitpunkten geschehen. Daher sollten die Autoren alle kritischen Zeitpunkte und die Anpassungsregeln beschreiben, die an diesen Zeitpunkten gelten (Beispiel 9d). Wenn Entscheidungshilfen oder Anleitungen benutzt werden, wie z.B. Flowcharts, Algorithmen oder Dosiertabellen, sollten diese abgebildet oder referenziert werden (Beispiel 9d), oder es sollte angegeben werden, wo man sie findet (Beispiel 9a).

\section{Item 10. Modifikation: Wenn die Intervention im} Verlauf der Studie modifiziert wurde, die Änderungen beschreiben (was, warum, wann und wie).

Beispiele

- 10a. In dieser Studie wurden 95\% der Screening- und kurzen Interventionsaktivitäten von Allgemeinmedizinern und Pflegefachkräften durchgeführt. ... aufgrund der verzögerten Rekrutierung unterstützten die wissenschaftlichen Mitarbeiter, die das Training zur Studie durchgeführt hatten, das Screening und die Kurzintervention in 10 Praxen und rekrutierten 152 Patienten, was $5 \%$ aller Studienteilnehmer entsprach.

- 10b. Aufgrund von Computern mit langsamen Prozessoren und schlechten Internetverbindungen stand 7 Allgemeinmediziner keine funktionale Software zur Verfügung. Sie nutzten eine strukturierte Papierversion, die zwischen Forscherteam und Allgemeinmedizinern nach jedem Termin per Fax übermittelt wurde.

\section{Erklärung}

Dieses Item berichtet über Modifikationen, die auf der Ebene der Studie durchgeführt werden, und nicht über individuelle Anpassungen wie in Item 9 beschrieben. Unvorhergesehene Modifikationen der Intervention können im Verlauf einer Studie auftreten, insbesondere bei Studien früher Phasen. Falls das passiert, ist es wichtig zu erklären, was modifiziert wurde, warum und wann die Modifikationen auftraten und wie die modifizierte In- tervention sich vom Original unterschied (Beispiel 10a - modifiziert wer intervenierte; Beispiel 10b - Materialien modifiziert). Manchmal sind Modifikationen eine Reaktion auf geänderte Rahmenbedingungen. In anderen Studien können sie jedoch auch einen Lernvorgang hinsichtlich der Intervention aufzeigen, dessen Weitergabe an Leser und weitere Personen wichtig ist, um bei einer späteren Replikation der Intervention unnötige Wiederholungen von Fehlern zu vermeiden. Wenn Änderungen der Intervention zwischen der Publikation von Protokoll oder Pilotstudie und der Primärpublikation auftraten, sollten diese Änderungen auch beschrieben werden.

Item 11. Wie gut (geplant): Wenn für die Intervention die Adhärenz oder die Therapietreue beurteilt wurden, beschreiben wie und von wem; falls Strategien eingesetzt wurden, um die Therapietreue zu erhalten oder zu verbessern, auch diese beschreiben.

Beispiele

- 11a. Pathologen wurden darin geschult, die laterale Tumorausbreitung gemäß Protokoll zu identifizieren [Referenz]. Die histopathologischen Untersuchungsergebnisse der Proben wurden durch ein Gremium von Referenz-Pathologen und Qualitätsmanager überprüft.

- 11b. Zu Beginn wurden die Mitarbeiter in den Studienzentren geschult und die Therapiesupervision erfolgte durch wöchentliche Treffen zwischen Therapeuten und Untersuchern. Die Sitzungen der kognitiven Therapie wurden mit Zustimmung der Teilnehmer auf Tonband aufgenommen. Dadurch konnten die Teilnehmer gebeten werden, die Aufnahmen als Teil ihrer Hausaufgabe anzuhören und die Supervision zu unterstützen. Im Verlauf der Studie wurde eine Stichprobe aus 80 Aufnahmen mit der revidierten Skala für kognitive Therapie [Referenz] und der Skala für Adhärenz zur kognitiven Therapie bei Risikogruppen [Referenz] bewertet, um eine strikte Beachtung des Protokolls während der gesamten Studie sicherzustellen. Diese Aufnahmen wurden sowohl aus frühen als auch späten Phasen der Therapie gezogen und umfassten Teilnehmer aus jedem Rekrutierungsjahr.

- 11c. Die Einhaltung der Studienmedikation wurde durch Mittelwerte selbst-berichteter Tablettenzählungen evaluiert, die während der telefonischen Kontakte ermittelt wurden. Diese Daten wurden kategorisiert in keine Tabletten eingenommen, fast keine Tabletten eingenommen (1-24\% der vorgeschriebenen Dosis), einige Tabletten eingenommen (25-49\%), die meisten Tabletten eingenommen (50-74\%), oder alle Tabletten eingenommen (75-100\%).

- 11d. Das Training wird in jedem der 3 regionalen Studienzentren voneinander unabhängig angeboten werden. Um über die Zentren hinweg eine standardisierte Erbringung des Trainings sicherzustellen, werden alle Trainer dasselbe Trainingsprotokoll befolgen. Die Durchführung des Trainings wird von allen Trainern gemeinsam geplant und eingeübt unter Verwendung von Rollenspielen und Peer-Review-Techniken. Zusätzlich wird der Projektleiter in jedem Zentrum während der ersten beiden Trainingssitzungen als Beobachter fungieren und den Trainern Rückmeldung geben hinsichtlich einer weiteren Standardisierung des Trainings [zu beachten: dieses Beispiel entstammt einem Protokoll].

\section{Erklärung}

Therapietreue bezieht sich auf den Grad, zu welchem eine Intervention so erfolgt, wie die Forscher sie geplant haben [20], und 
kann den Erfolg der Intervention beeinflussen [21]. Die Begriffe, die zur Beschreibung dieses Konzepts verwendet werden, variieren zwischen den Disziplinen und umfassen Behandlungsintegrität, Adhärenz der Behandler oder Teilnehmer und Implementationstreue. Dieses Item - sowie auch Item 12 - geht weit über den reinen Erhalt der Intervention hinaus (z.B. wie viele Teilnehmer wurden mit dem Medikament oder der Trainings-Intervention behandelt) und bezieht sich darauf, „wie gut“ die Intervention angenommen oder durchgeführt wurde (z.B. wie viele Teilnehmer nahmen das Medikament ein/führten die Übung durch, wie oft nahmen sie es ein/führten sie aus und für wie lange). Abhängig von der Intervention kann Therapietreue einen oder mehrere Teile der Intervention betreffen, so z.B. das Training der Behandelnden (Beispiele 11a, 11b, 11d), die Durchführung der Intervention (Beispiel 11b) und die Annahme der Intervention (Beispiel 11c). Die Messungen zur Evaluation der Interventionstreue werden ebenfalls in Abhängigkeit vom Interventionstyp variieren. Z.B. konzentriert sich die Bewertung der Therapietreue bei einfachen pharmakologischen Interventionen oftmals auf die Befolgung der Medikamenteneinnahme durch die Empfänger (Beispiel 11b). Bei komplexen Interventionen, wie z. B. Rehabilitation, psychologischen Behandlungen oder Interventionen zur Verhaltensänderung, ist die Bewertung der Therapietreue jedoch komplexer (Beispiel 11b). Es gibt verschiedene etablierte Strategien und Werkzeuge, die vor der Durchführung der Intervention (Beispiel 11d) oder während der Studie (Beispiel 11b) genutzt werden können, um die Therapietreue sicherzustellen. Falls Strategien oder Werkzeuge eingesetzt wurden, um die Therapietreue sicherzustellen, sollten diese eindeutig beschrieben werden. Jegliche Materialien zur Bewertung oder Aufrechterhaltung der Therapietreue sollten beschrieben, referenziert oder ihre Quelle angegeben werden.

\section{Item 12. Wie gut (tatsächlich): Wenn für die}

Intervention die Adhärenz oder die Therapietreue bewertet wurde, beschreiben in welchem Ausmaß die Intervention wie geplant ausgeführt wurde.

\section{Beispiele}

- 12a. Die durchschnittliche Anzahl (SD) der besuchten Physiotherapiesitzungen war 7,5 (1,9). 7 Patienten (9\%) beendeten weniger als 4 Physiotherapiesitzungen; die Gründe umfassen Nichterscheinen, Umzug in einen anderen Bundesstaat oder Abklingen der Schmerzsymptomatik. In mindestens 5 der 7 Wochen befolgten $70 \%$ der Patienten in der Physiotherapiegruppe ihr häusliches Übungsprogramm.

- 12b. Die FÜ-Gruppe [frühe Übung] berichtete einen Einhaltungsgrad von $73 \%$ zum Zeitpunkt [Zeitangabe] T2 und 75,7\% zum Zeitpunkt [Zeitangabe] T3, und die VÜ-Gruppe [verzögerte Übung] berichtete 86,7\% Einhaltung zum Zeitpunkt T3 ... wobei die frühe Übungsgruppe (FÜ) von krankheits- und behandlungsbezogenen Trainingshindernissen während ihrer Krebsbehandlung („Woche der Chemotherapie“ $14 \%$, ermüdet $10 \%$ ) oder von lebensbezogenen Hindernissen („Krankheit, z.B. Erkältungen oder Grippe“ 16\%, familiäre Verpflichtungen $13 \%$ ) berichteten.

- 12c. Insgesamt 214 Teilnehmer (78\%) berichteten, dass sie mindestens $75 \%$ der Studientabletten eingenommen hatten; der Anteil der Patienten, die berichteten, dass sie mindesten $75 \%$ der Tabletten eingenommen hatten, war in beiden Gruppen ähnlich.

- 12d. Die Integrität der psychologischen Therapie wurde mit der Bewertungsskala zur Kognitiven Therapie [Referenz] an- hand von Transkripten aus 40 Onlinesitzungen von Patienten, die mindestens 5 Therapiesitzungen beendet hatten, bewertet. Für jeden Therapeuten wurde mindestens ein solcher Patient mittels computergenerierter Zufallszahlen ausgewählt. Für diese Patienten wurde entweder die sechste oder die vorletzte Sitzung durch 2 unabhängige, in KVT [kognitive Verhaltenstherapie] ausgebildete Psychologen bewertet; diese bewerteten im Mittel mit 31 (SD zwischen den Therapeuten: 9) und 32 (13) von 72 Punkten.

\section{Erklärung}

Aus verschiedenen Gründen kann eine Intervention, oder Teile von ihr, möglicherweise nicht so durchgeführt werden, wie geplant und dies kann Auswirkungen auf die Therapietreue bei der Durchführung der Intervention haben. Falls diese bewertet wird, sollten Autoren das Ausmaß beschreiben, zu dem sich die durchgeführte von der geplanten Intervention unterschied. Diese Information kann helfen, Studienergebnisse zu erklären, Fehler bei der Interpretation der Studienergebnisse zu minimieren, auf zukünftige Modifikationen der Interventionen hinzuweisen und - wenn die Therapietreue niedrig ist - den Bedarf an weiteren Untersuchungen oder Strategien zur Verbesserung der Therapietreue oder Adhärenz aufzuzeigen. [22,23] Z. B. könnte es manche Aspekte der Intervention geben, denen sich die Teilnehmer nicht unterziehen möchten und dies könnte ihre Adhärenz beeinflussen. Der Bericht zur Interventionstreue wird sich auf die Messungen beziehen, die zur Bewertung herangezogen und in Item 11 beschrieben wurden (Beispiele 12a-d).

\section{Diskussion}

Wer sollte TIDieR nutzen?

Wir beschreiben eine kurze Liste von Items, die nach unserer Auffassung dazu genutzt werden kann, die Berichterstattung von Interventionen zu verbessern. Sie erleichtert es Autoren, Forschungsberichte über ihre Interventionen zu strukturieren, Gutachtern und Herausgebern, diese Beschreibungen zu bewerten, und Lesern, die verbesserte Information zu nutzen. In Übereinstimmung mit dem CONSORT 2010 und dem SPIRIT 2013 Statement empfehlen auch wir, dass Interventionen so detailliert beschrieben werden, dass eine Replikation möglich ist, und Autoren hierfür die TIDieR Checkliste nutzen. Da die Beschreibung aller Details der Interventionen in der Primärpublikation einer Studie nicht immer möglich ist, empfiehlt die TIDieR Checkliste den Autoren, darauf hinzuweisen, dass jedes Item beschrieben ist und anzugeben, wo diese detaillierten Informationen hinterlegt sind.

Die Anzahl der berichteten Checklisten-Items verbessert sich, wenn Zeitschriften im Rahmen der Artikeleinreichung die Anwendung einer Checkliste fordern. [24] Wir ermutigen Zeitschriften, die Anwendung der TIDieR Checkliste in gleicher Weise zu unterstützen wie das CONSORT Statement und ähnliche Leitlinien zur Berichterstattung. Dies kann durch eine Anpassung der Hinweise für Autoren geschehen, durch Publikation eines Editorials zum Berichten von Interventionen und ein Verlinken der Checkliste auf der Internetseite der Zeitschrift. Derzeit bieten nur wenige Zeitschriften spezifische Anleitungen zum Berichten von Interventionen an [25]. Auch vertreten nur wenige die Herausgeberpolitik, dass sie Studien nur dann veröffentlichen, wenn Interventionsprotokolle oder ausführliche Details zur Verfügung gestellt werden. [26] Wir ermutigen weitere 
Zeitschriften, eine ähnliche Herausgeberpolitik in Erwägung zu ziehen. Jegliche von Zeitschriften und Autoren angegebenen Links sollten zuverlässig und dauerhaft sein. Für die Interventionsbeschreibungen sind ebenso dauerhafte Hinterlegungsstellen notwendig. Deren Bereitstellung erfordert die Zusammenarbeit aller Akteure in der Forschung (z.B. Forscher, Herausgeber, Verlage und Forschungsförderer).

Autoren könnten TIDieR Items auch zur Interventionsbeschreibung in systematischen Übersichtsarbeiten nutzen, sodass Leser Zugang zu allen Details aller Interventionen haben (oder zumindest wissen, wo detailliertere Informationen zu finden sind), die sie nach dem Lesen der Übersichtsarbeit replizieren wollen.

\section{TIDieR in Kombination mit dem CONSORT und SPIRIT Statement nutzen}

Wir empfehlen Autoren, die Berichte zu randomisierten kontrollierten Studien einreichen, TIDieR gemeinsam mit der CONSORT Checkliste zu nutzen. Die Autoren sollten bei Item 5 der CONSORT Checkliste auf TIDieR verweisen und eine separat ausgefüllte TIDieR Checkliste zur Verfügung stellen. Zeitschriften, die beabsichtigen, unsere Empfehlungen umzusetzen, sollten die Hinweise für ihre Autoren entsprechend ändern sowie ihre Herausgeber und Gutachter darüber in Kenntnis setzen. Desgleichen kann die TIDieR Checkliste bei der Einreichung von Studienprotokollen zur Bearbeitung von Item 11 der SPIRIT 2013 Checkliste eingesetzt werden. Jedoch treffen 2 der TIDieR Items (Items 10 und 12) bei Protokollen nicht zu, da sie erst nach Beendigung der Studie relevant sind. Dies ist in der TIDieR Checkliste vermerkt. Wahrscheinlich wird die Bedeutung publizierter Protokolle als Informationsquelle für Interventionsbeschreibungen zunehmen und kann durch die kombinierte Nutzung von TIDieR und SPIRIT 2013 unterstützt werden. Für die Publikation anderer, nicht randomisiert kontrollierter Studiendesigns kann TIDieR als alleinige Checkliste oder in Kombination mit einem anderen für das jeweilige Design relevante Statement genutzt werden (z.B. gemeinsam mit dem STROBE Statement [12]). Wir sind uns bewusst, dass eine gute Beschreibung komplexer Interventionen eine Herausforderung darstellt. Eine Checkliste wie TIDieR könnte hier die Interventionsbeschreibung teilweise unterstützen, möglicherweise jedoch nicht ihre volle Komplexität erfassen.

Es ist uns auch bewusst, dass die Anwendung der TIDieR Checkliste zu einer erhöhten Anzahl von Wörtern bei Studienpublikationen führen kann, insbesondere wenn die Protokolle der Studien nicht veröffentlicht wurden. Nach unserer Auffassung ist eine erhöhte Anzahl von Wörtern jedoch notwendig, um die Berichterstattung von Studien im Allgemeinen und die Beschreibungen von Interventionen im Speziellen zu verbessern. Je mehr Zeitschriften die Bedeutung gut berichteter Studien und umfassend beschriebener Methoden erkennen und zu einem reinen oder gemischten Online-Publikationsmodell mit Verlinkung zum Studienprotokoll wechseln, desto weniger wird die Zahl der Wörter eine Barriere für die Qualität der Berichterstattung darstellen. Bspw. nahm die Nature Publishing Group für den Methodenabschnitt die Begrenzung für die Anzahl der Wörter zurück und gab folgende Instruktion: „Wenn für eine komplette Methodenbeschreibung mehr Wörter notwendig sind, sollte der Autor eine 300 Wörter umfassende Methodenzusammenfassung erstellen und einen zusätzlichen Methodenteil am Ende des Textes nach den Legenden für die Abbildungen einfügen. Dieser Methodenteil wird in der Online-Version, nicht aber in der gedruckten
Version des Artikels erscheinen. Der Methodenteil sollte so konzise wie möglich geschrieben werden, aber alle Elemente enthalten, die für eine Replikation und Interpretation der Ergebnisse notwendig sind [27].“

\section{Schlussfolgerung}

Die TIDieR Checkliste und Anleitung soll Autoren, Herausgeber, Gutachter und Leser unterstützen. Manche Autoren könnten die Checkliste als eine weitere zeitaufwendige Hürde empfinden und sich für die Publikation in Zeitschriften entscheiden, die nicht die Anwendung von Leitlinien zur Berichterstattung fordern. Jedoch zeigen wissenschaftliche Untersuchungen eindeutig, dass die Qualität der Berichterstattung in der Gesundheitsforschung inakzeptabel schlecht ist. Durch entsprechende Unterstützung und gute Implementation von Leitlinien für Forschungsberichte können Verlage, Herausgeber, Gutachter und Autoren ihrer Aufgabe gerecht werden, dafür zu sorgen, dass vollständig und transparent berichtet wird, was genau getan und gefunden wurde [28]. So wird unnötige Forschung reduziert $[29,30]$ und ihr Potenzial, Gesundheit zu verbessern, gestärkt.

\section{Danksagung}

Wir danken allen Teilnehmern der Delphi Umfrage für ihre kritischen Kommentare. Ebenso geht unser Dank an Nicola Pidduck (Department of Primary Care Health Sciences, Oxford University) für ihre Unterstützung in der Organisation der Konsensuskonferenz in Oxford.

Beitragende: PPG und TCH initiierten die TIDieR Gruppe und leiteten die Organisation der Delphi Umfragen und der Konsensuskonferenz in Verbindung mit anderen Mitgliedern des Lenkungsausschusses (IB, RM und RP). TCH leitete die Erstellung des Manuskripts. Alle Autoren trugen zum Entwurf und zur Revision des Manuskripts bei und genehmigten die finale Version. TCH und PPG sind die Hauptverantwortlichen.

Finanzierung: Für die Entwicklung der Checkliste und Anleitung gab es keine explizite Finanzierung. Die Konsensuskonferenz im März 2013 wurde teilweise finanziert durch einen NIHR Senior Investigator Award, den PPG erhielt. TCH wird unterstützt vom National Health and Medical Research Council of Australia (NHMRC)/Primary Health Care Research Evaluation and Development Career Development Fellowship (1033038), der Mittel vom Australian Department of Health and Ageing erhält. PPG wird unterstützt vom NHMRC Australia Fellowship (527500). DGA wird unterstützt durch ein Förderprogramm des Cancer Research UK (C5529). MDW wird unterstützt vom Wellcome Trust Senior Investigator award (WT097899MA).

Interessenskonflikte: Alle Autoren haben das "Unified Competing Interest form” (www.icmje.org/coi_disclosure.pdf, auf Anfrage von der korrespondierenden Autorin erhältlich) ausgefüllt und folgendes erklärt: RM ist angestellt beim NETSCC, Teil des National Institute for Health Research (NIHR) in England. Das NETSCC leitet die NIHR Journals Library im Auftrag des NIHR, "eine Gruppe von 5 Open-Access-Zeitschriften, die ein wichtiges und dauerhaftes Archiv für die vom National Institute for Health Research finanzierte Forschung vorhält.“ Die NIHR Journals Library legt großen Wert auf das Berichten vollständiger Ergebnisse aus finanzierter Forschung und wird TIDieR voraussichtlich 
genauso nutzen wie die anderen Leitlinien zur Berichterstattung. VB war zum Zeitpunkt der Konsensuskonferenz und der initialen Manuskripterstellung Chefherausgeber von PLOS Medicine. HM ist Mitherausgeber des BMJ, war aber an keiner Entscheidung hinsichtlich des Manuskripts beteiligt.

Herkunft und Begutachtung: Nicht beauftragt, extern begutachtet.

\section{Kernaussagen}

Ohne vollständig publizierte Interventionsbeschreibungen können Kliniker und Patienten effektive Interventionen nicht zuverlässig umsetzen.

In publizierten Studien ist die Qualität der Interventionsbeschreibungen bemerkenswert schlecht, unabhängig vom Typus der Intervention.

Die Checkliste und der Leitfaden zur Interventionsbeschreibung und Replikation (TIDieR) sind entwickelt worden, um die Vollständigkeit der Berichterstattung von Interventionen und damit letztlich ihre Replizierbarkeit zu verbessern.

TIDieR kann von Autoren genutzt werden, um das Berichten ihrer Interventionen zu strukturieren, von Gutachtern und Herausgebern, um die Vollständigkeit der Berichterstattung zu bewerten, und von Lesern, die diese Information nutzen möchten.

Autoren

T. C. Hoffmann ${ }^{1}$ associate professor of clinical epidemiology,

P. P. Glasziou ${ }^{1}$ director and professor of evidence based medicine,

I. Boutron ${ }^{2}$ professor of epidemiology,

R. Milne ${ }^{3}$ professorial fellow in public health and director,

R. Perera ${ }^{4}$ university lecturer in medical statistics,

D. Moher ${ }^{5}$ senior scientist,

D. G. Altman ${ }^{6}$ Douglas G Altman professor of statistics in medicine,

V. Barbour ${ }^{7}$ medicine editorial director, PLOS,

H. Macdonald ${ }^{8}$ assistant editor,

M. Johnston ${ }^{9}$ emeritus professor of health psychology,

S. E. Lamb ${ }^{10}$ Kadoorie professor of trauma rehabilitation and co-director of Oxford clinical trials research unit,

M. Dixon-Woods ${ }^{11}$ professor of medical sociology,

P. McCulloch ${ }^{12}$ clinical reader in surgery,

J. C. Wyatt ${ }^{13}$ leadership chair of ehealth research,

A.-W. Chan ${ }^{14}$ phelan scientist,

S. Michie ${ }^{15}$ professor

\section{Institute}

Centre for Research in Evidence Based Practice, Faculty of Health Sciences and Medicine, Bond University, Queensland, Australia, 4229

INSERMU738, Université Paris Descartes-Sorbonne Paris Cité, Paris, France

${ }^{3}$ Wessex Institute, University of Southampton, Southampton, UK

${ }^{4}$ Department of Primary Care Health Sciences, University of Oxford, UK

${ }^{5}$ Clinical Epidemiology Program, Ottawa Hospital Research Institute,

Ottawa, Canada

Centre for Statistics in Medicine, University of Oxford, UK

7 PLOS, Brisbane, Australia

BMJ, London, UK

${ }^{9}$ Institute of Applied Health Sciences, University of Aberdeen, Aberdeen, UK

${ }^{10}$ Nuffield Department of Orthopaedics, Rheumatology and Musculoskeletal

Sciences, Botnar Research Centre, University of Oxford, Oxford, UK

${ }^{1}$ Department of Health Sciences, University of Leicester, Leicester, UK

${ }^{12}$ Nuffield Department of Surgical Science, University of Oxford, Oxford, UK

${ }^{13}$ Leeds Institute of Health Sciences, University of Leeds, Leeds, UK

${ }^{14}$ Women's College Research Institute, University of Toronto, Toronto,

Canada

${ }^{5}$ Centre for Outcomes Research and Effectiveness, Department of Clinical,

Educational and Health Psychology, University College London, London, UK

\section{Literatur}

1 Duff J, Leather H, Walden E et al. Adequacy of published oncology randomised controlled trials to provide therapeutic details needed for clinical application. J Natl Cancer Inst 2010; 102: 702-705

2 Glasziou P, Meats E, Heneghan C et al. What is missing from descriptions of treatment in trials and reviews? BMJ 2008; 336: 1472-1474

3 Hoffmann T, Erueti C, Glasziou P. Poor description of non-pharmacological interventions: analysis of consecutive sample of randomised trials. BMJ 2013; 347: f3755

4 Schulz K, Altman D, Moher D.CONSORT Group. CONSORT 2010 Statement: updated guidelines for reporting parallel group randomised trials. BMJ 2010; 340: c332

5 Schroter S, Glasziou P, Heneghan C. Quality of descriptions of treatments: a review of published randomised controlled trials. BMJ Open 2012; 2: e001978

6 Boutron I, Moher D, Altman D et al. Extending the CONSORT statement to randomised trials of nonpharmacologic treatment: explanation and elaboration. Ann Intern Med 2008; 148: 295-310

7 MacPherson H, Altman DG, Hammerschlag $R$ et al. Revised standards for reporting interventions in clinical trials of acupuncture (STRICTA): extending the CONSORT statement. PLoS Med 2010; 7: e1000261

8 Gagnier J, Boon H, Rochon $P$ et al. Reporting randomised, controlled trials of herbal interventions: an elaborated CONSORT statement. Ann Intern Med 2006; 144: 364-367

9 Chan A, Tetzlaff J, Gøtzsche P et al. SPIRIT 2013 explanation and elaboration: guidance for protocols of clinical trials. BMJ 2013; 346: e7586

10 Moher D, Schulz K, Simera I et al. Guidance for developers of health research reporting guidelines. PLoS Med 2010; 7: e1000217

11 Murphy M, Black N, Lamping D et al. Consensus development methods, and their use in clinical guideline development. Health Technol Assess 1998; 2: 1-88

12 Von Elm E, Altman D, Egger $M$ et al. The Strengthening the Reporting of Observational Studies in Epidemiology (STROBE) statement: guidelines for reporting observational studies. BMJ 2007; 335: 806-808

13 De Bruin $M$, Viechtbauer $W$, Hospers $H$ et al. Standard care quality determines treatment outcomes in control groups of HAART-adherence intervention studies: implications for the interpretation and comparison of intervention effects. Health Psychol 2009; 28: 668-674

14 Thorpe K, Zwarenstein M, Oxman $A D$ et al. A pragmatic-explanatory continuum indicator summary (PRECIS): a tool to help trial designers. J Clin Epidemiol 2009; 62: 464-475

15 Craig P, Dieppe P, Macintyre S et al. Developing and evaluating complex interventions: the new Medical Research Council guidance. BMJ 2008; 337: a1655

16 McCleary N, Duncan E, Stewart F et al. Active ingredients are reported more often for pharmacologic than non-pharmacologic interventions: an illustrative review of reporting practices in titles and abstracts. Trials 2013; 14: 146

17 Michie $S$, West $R$. Behaviour change theory and evidence: a presentation to Government. Health Psychol Rev 2013; 7: 1-22

18 Dixon-Woods $M$, Leslie $M$, Tarrant $C$ et al. Explaining Matching Michigan: an ethnographic study of a patient safety program. Implement Sci 2013; 8: 70

19 Dixon-Woods $M$, Bosk C, Aveling E et al. Explaining Michigan: developing an ex post theory of a quality improvement program. Milbank Q 2011; 89: 167-205

20 Carroll $C$, Patterson $M$, Wood S et al. A conceptual framework for implementation fidelity. Implement Sci 2007; 2: 40

21 Bellg AJ, Borrelli B, Resnick $B$ et al. Enhancing treatment fidelity in health behaviour change studies: best practices and recommendations from the NIH Behaviour Change Consortium. Health Psychol 2004; 23: 443-451

22 Hardeman $W$, Michie S, Fanshawe T et al. Fidelity of delivery of a physical activity intervention: predictors and consequences. Psychol Health 2008; 23: 11-24

23 Spillane V, Byrne M, Byrne $M$ et al. Monitoring treatment fidelity in a randomised controlled trial of a complex intervention. J Adv Nursing 2007; 60: 343-352

24 Hopewell S, Ravaud P, Baron $G$ et al. Effect of editors' implementation of CONSORT guidelines on the reporting of abstracts in high impact medical journals: interrupted time series analysis. BMJ 2012; 344 : e4178

25 Hoffmann T, English T, Glasziou P. Reporting of interventions in randomised trials: an audit of journal Instructions to Authors. Trials 2014; 15: 20 
26 Michie S, Fixsen D, Grimshaw JM et al. Specifying and reporting complex behaviour change interventions: the need for a scientific method. Implement Sci 2009; 4: 40

27 Nature. For authors: manuscript formatting guide.[Internet] www. nature.com/nature/uthors/gta/index.html\#a5.3.

28 Turner L, Shamseer L, Altman DG et al. Does use of the CONSORT Statement impact the completeness of reporting of randomised controlled trials published in medical journals? A Cochrane review. Syst Rev 2012; 1: 60
29 Chalmers I, Glasziou P. Avoidable waste in the production and reporting of research evidence. Lancet 2009; 374: 86-89

30 Glasziou P, Altman D, Bossuyt $P$ et al. Reducing waste from incomplete or unusable reports of biomedical research. Lancet 2014; 383: 267-276
Hinweis

Dieser Artikel wurde gemäß des Erratums vom 27.12.2017 geändert.

\section{Erratum}

Der Autorenname A.-W. Chan war falsch und wurde korrigiert. 